\title{
Collisional redistribution of intense phase-fluctuation radiation
}

\author{
H F Arnoldus and G Nienhuis \\ Fysisch Laboratorium, Rijksuniversiteit Utrecht, Postbus 80000,3508 TA Utrecht, The \\ Netherlands
}

Received 14 December 1982, in final form 1 March 1983

\begin{abstract}
We incorporate in the theory of collisional redistribution of resonance radiation the effect of a finite bandwidth of the intense incident radiation. The phase of the radiation is treated as a process with independent increments, which contains the phase-diffusion model as a special case. These phase fluctuations give rise to effective decay operators affecting the evolution of the density matrix and the regression of the correlation function. For a two-level atom suffering binary collisions the combined effect of collisions and phase fluctuations on the fluorescence spectrum is evaluated in several limiting cases of practical interest.
\end{abstract}

\section{Introduction}

The response of atomic systems to intense and stochastically fluctuating radiation fields near resonance is of practical importance since the availability of tunable laser sources. The processes studied include multiphoton ionisation (Zoller 1979a, 1982), AC Stark splitting in double optical resonance (Zoller 1979b, Georges and Lambropoulos 1979, Zoller et al 1981), two-photon absorption (Salomaa 1978) and in particular resonance fluorescence (Agarwal 1976, 1978, Eberly 1976, Kimble and Mandel 1977, Avan and Cohen-Tannoudji 1977, Zoller 1977, 1978a, b, Knight et al 1978, Wódkiewicz 1980, Chaturvedi and Gardiner 1981, Georges and Dixit 1981). The laser models studied most extensively include the phase-diffusion model (Agarwal 1976, 1978, Kimble and Mandel 1977, Zoller 1977, 1978a, Wódkiewicz 1980), which is expected to give a fair representation of an intensity-stabilised single-mode laser well above threshold, and the chaotic field with Gaussian amplitude fluctuations (Zoller $1979 \mathrm{a}, \mathrm{b}, \mathrm{Georges}$ and Lambropoulos 1979), which provides a reasonable description of a multimode laser.

Another line of research during the last decade concerned the collisional redistribution of monochromatic radiation, both for low (Omont et al 1972, Nienhuis and Schuller 1977, Burnett and Cooper 1980) and high (Burnett et al 1982, Nienhuis 1982, Reynaud and Cohen-Tannoudji 1982) intensities.

In the present paper we study the combined effect of collisions and laser fluctuations on the spectrum of resonance fluorescence. This problem is important in order to disentangle the collisional effects from the observed total fluorescence spectrum excited by intense laser radiation, which in general is not quite monochromatic. At the same time this study enables us to compare both types of broadening effects. As a model for the phase fluctuations of the incident radiation we take a jump-like Markov process, 
which has the phase-diffusion model as a Gaussian limit. The effect of these fluctuations on the evolution of the average density matrix of the particle system, and on the regression of the average dipole correlation function can be expressed in terms of equivalent Liouville operators containing additional effective damping operators. Previous results (Nienhuis 1982) for the fluorescence spectrum in the binary-collision approximation can then be directly applied to the present problem, giving rise to simple expressions for the widths and the strengths of the lines in the fluorescence spectrum in special limiting cases.

\section{General expression for the fluorescence spectrum}

A single active atom in a bath of $N$ perturbers is irradiated with phase-fluctuating light with central frequency $\omega_{\mathrm{L}}$, polarisation $\varepsilon_{\mathrm{L}}$ and amplitude $E_{0}$. The radiation drives the transition between the ground level ( $g$ ) and an excited level (e) of the atom, which may both consist of several degenerate or nearly degenerate substates. Coupling with other levels is ignored, and we neglect collisional coupling between the ground level and the excited level.

The evolution of the density matrix $\rho(t)$ of the entire particle system is governed by the Liouville equation

$$
\frac{\mathrm{d}}{\mathrm{d} t} \rho(t)=-\frac{\mathrm{i}}{\hbar}[H(t), \rho(t)]-\Gamma \rho(t)
$$

where $\Gamma$ denotes the effect of spontaneous emission of the atom, and where the full Hamiltonian is

$$
H(t)=H_{\mathrm{a}}+\sum_{i} H_{i}+H_{\mathrm{ar}}(t)
$$

with $H_{\mathrm{a}}$ the Hamiltonian of the free atom, and $H_{i}$ the Hamiltonian of the $i$ th perturber, including its interaction with the atom. Interactions between perturbers are ignored. The interaction $H_{\text {ar }}$ between the atom and the classical radiation field in the rotatingwave approximation is expressed as

$$
H_{\mathrm{ar}}(t)=-\frac{1}{2} d_{\mathrm{L}} E_{0} \exp \left(-\mathrm{i} \omega_{\mathrm{L}} t-\mathrm{i} \psi(t)\right)+\text { Hermitian conjugate }
$$

where $d_{\mathrm{L}}=\boldsymbol{\varepsilon}_{\mathrm{L}} \cdot \boldsymbol{\mu}^{(+)}$is the component of the raising part of the atomic dipole in the polarisation direction. The phase $\psi(t)$ of the radiation field will be treated as a stochastic process, which turns the Liouville equation (2.1) into a stochastic differential equation. We denote as $T\left(t, t^{\prime}\right)$ the evolution operator that transforms $\rho\left(t^{\prime}\right)$ into $\rho(t)$

$$
\rho(t)=T\left(t, t^{\prime}\right) \rho\left(t^{\prime}\right)
$$

so that $T$ obeys the same Liouville equation as $\rho$, with the initial condition $T\left(t^{\prime}, t^{\prime}\right)=1$.

We wish to eliminate the rapid oscillations with the optical frequency $\omega_{\mathrm{L}}$, by the stochastic transformation

$$
\rho(t)=\exp \left[-\mathrm{i}\left(\omega_{\mathrm{L}} t+\psi(t)\right) K\right] \sigma(t)
$$

with

$$
K \sigma=\frac{1}{2}\left[P_{\mathrm{e}}-P_{\mathrm{g}}, \sigma\right]=\left[P_{\mathrm{e}}, \sigma\right]
$$

where $P_{\mathrm{e}}$ and $P_{\mathrm{g}}$ are the projection operators on the substates of the excited level 
and the ground level. The evolution operator $U\left(t, t^{\prime}\right)$ that transforms $\sigma\left(t^{\prime}\right)$ into $\sigma(t)$ obeys the effective Liouville equation

$$
\frac{\mathrm{d}}{\mathrm{d} t} U\left(t, t^{\prime}\right)=\left(-\mathrm{i} L_{\mathrm{d}}-\mathrm{i} \sum_{i} L_{\mathrm{i}}-\Gamma+\mathrm{i} \dot{\psi}(t) K\right) U\left(t, t^{\prime}\right)
$$

where $L_{i}$ indicates a commutator with $H_{i}$, and $L_{\mathrm{d}}$ is a commutator with an effective (dressed-atom) Hamiltonian

$$
-\mathrm{i} L_{\mathrm{d}} \sigma=-\frac{\mathrm{i}}{\hbar}\left[H_{\mathrm{d}}, \sigma\right]=-\frac{\mathrm{i}}{\hbar}\left[H_{\mathrm{a}}-\frac{1}{2} d_{\mathrm{L}} E_{0}-\frac{1}{2} \mathrm{~d}_{\mathrm{L}}^{\dagger} E_{0}, \sigma\right]+\mathrm{i} \omega_{\mathrm{L}} K \sigma
$$

The spectrum of resonance fluorescence with polarisation $\varepsilon$ is proportional to the Fourier-Laplace transform of the autocorrelation function of $d=\mu^{(+)} \cdot \boldsymbol{\varepsilon}$. We can express this correlation function in terms of the transformed density matrix and evolution operator by applying the identity

$$
\operatorname{Tr} d^{\dagger} T(t, 0)(\rho(0) d)=\exp \left(-\mathrm{i} \omega_{\mathrm{L}} t\right) \operatorname{Tr} d^{\dagger} U_{\mathrm{r}}(t, 0)(\sigma(0) d)
$$

where the evolution operator $U_{\mathrm{r}}$ that determines the regression of the correlation function is related to the evolution operator $U$ by the equation

$$
U_{\mathrm{r}}\left(t, t^{\prime}\right)=\exp \left(-\mathrm{i} \psi(t)+\mathrm{i} \psi\left(t^{\prime}\right)\right) U\left(t, t^{\prime}\right)
$$

We conclude that the spectral power of the fluorescence with polarisation $\varepsilon$ emitted per unit solid angle is given by the formal expression

$$
I(\omega)=\frac{a}{\pi} \operatorname{Re} \int_{0}^{\infty} \mathrm{d} t \exp \left[\mathrm{i}\left(\omega-\omega_{\mathrm{L}}\right) t\right]\left\langle\operatorname{Tr} d^{+} U_{\mathrm{r}}(t, 0)(\sigma(0) d)\right\rangle
$$

where $a=\omega^{4} / 8 \pi^{2} \varepsilon_{0} c^{3}$, and where the angle brackets denote an average over the probability distribution of the stochastic phase. It is worth mentioning that the regression operator $U_{\mathrm{r}}$ obeys the Liouville equation

$$
\frac{\mathrm{d}}{\mathrm{d} t} U_{\mathrm{r}}\left(t, t^{\prime}\right)=\left(-\mathrm{i} L_{\mathrm{d}}-\mathrm{i} \sum_{i} L_{i}-\Gamma+\mathrm{i} \dot{\psi}(t)(K-1)\right) U_{\mathrm{r}}\left(t, t^{\prime}\right)
$$

which differs from the equation (2.7) for the evolution operator $U$ that describes the time behaviour of the density matrix $\sigma(t)$. Obviously both $\sigma(0)$ and $U_{\mathrm{r}}(t, 0)$ in $(2.11)$ are stochastic quantities, due to their dependence on the fluctuating phase.

In the next section we shall perform the stochastic average by adopting a specific model for the fluctuating phase.

\section{Stochastic average of evolution and regression}

We treat the phase of the incident radiation as a process with independent increments (Van Kampen 1981). This means that $\psi$ is a Markov process with a conditional probability $P\left(\psi, t \mid \psi^{\prime}, t^{\prime}\right)$ that depends only on the time interval $t-t^{\prime}$, and on the phase difference $\psi-\psi^{\prime}$. Then the transition probability per unit time from the phase $\psi^{\prime}$ to the phase $\psi$ must be a function $w(\eta)$ that is independent of time, and depends only on the phase increment $\eta=\psi-\psi^{\prime}$. The time-dependent probability distribution 
function $P(\psi, t)$ then obeys the master equation:

$$
\frac{\partial}{\partial t} P(\psi, t)=\int \mathrm{d} \eta w(\eta)(P(\psi-\eta, t)-P(\psi, t)) .
$$

This corresponds to the physical picture that the phase $\psi$ makes a random jump at independent random instants. The transition rate $w(\eta)$ is normalised to the frequency of the random instants, and its shape gives the distribution over the sizes of the jumps. The same model has been applied to free atoms by Zoller and Ehlotzky (1977).

We now consider an operator $V(t)$ that obeys the Liouville-type equation

$$
\frac{\mathrm{d}}{\mathrm{d} t} V(t)=\left(-\mathrm{i} L_{0}-\mathrm{i} \dot{\psi}(t) L_{1}\right) V(t)
$$

with an initial condition $V(0)=V_{0}$ that does not depend on the phase values $\psi(t)$ for $t>0$. In the appendix we prove that the stochastic average of $V$ obeys the nonstochastic differential equation

$$
\frac{\mathrm{d}}{\mathrm{d} t}\langle V(t)\rangle=\left(-\mathrm{i} L_{0}-W\right)\langle V(t)\rangle
$$

where $W$ is an effective decay operator defined by

$$
W=\int \mathrm{d} \eta w(\eta)\left[1-\exp \left(-\mathrm{i} \eta L_{1}\right)\right]
$$

Obviously the initial value of $\langle V\rangle$ is $\langle V(0)\rangle=\left\langle V_{0}\right\rangle$. With this initial condition equation (3.3) determines the stochastic average of $V(t)$ for all positive times.

We can apply this result directly to the operator $U_{\mathrm{r}}(t, 0)(\sigma(0) d)$ in $(2.11)$, since the initial density matrix $\sigma(0)$ at time zero cannot depend upon the stochastic phase $\psi(t)$ at positive times. Furthermore the same result can be used to obtain an effective evolution equation for the average density matrix $\langle\sigma(t)\rangle$, which in turn determines the average steady-state density matrix $\bar{\sigma}$. Hence the average correlation function in (2.11) can be expressed as

$$
\left\langle\operatorname{Tr} d^{+} U_{\mathrm{r}}(t, 0)(\sigma(0) d)\right\rangle=\operatorname{Tr} d^{\dagger} \bar{U}_{\mathrm{r}}(t, 0)(\bar{\sigma} d)
$$

where the average regression operator $\bar{U}_{\mathrm{r}}$ is determined by the effective Liouville equation

$$
\frac{\mathrm{d}}{\mathrm{d} t} \bar{U}_{\mathrm{r}}(t, 0)=\left(-\mathrm{i} L_{\mathrm{d}}-\mathrm{i} \sum_{i} L_{i}-\Gamma-W_{\mathrm{r}}\right) \bar{U}_{\mathrm{r}}(t, 0)
$$

with $W_{\mathrm{r}}$ given by equation (3.4) after substituting $1-K$ for $L_{1}$. The density matrix $\bar{\sigma}$ in $(3.5)$ is the steady-state limit of the solution of the effective evolution equation

$$
\frac{\mathrm{d}}{\mathrm{d} t}\langle\sigma(t)\rangle=\left(-\mathrm{i} L_{\mathrm{d}}-\mathrm{i} \sum_{i} L_{i}-\Gamma-W_{\mathrm{e}}\right)\langle\sigma(t)\rangle
$$

where now $W_{\mathrm{e}}$ is given by equation (3.4) after substitution of $-K$ for $L_{1}$.

The eigenvalues of $K$ are immediately obvious from the definition (2.6). It gives zero when operating on a density matrix that has no matrix element coupling the 
excited states and the ground states, it has eigenvalue 1 when operating on a part $P_{\mathrm{e}} \sigma P_{\mathrm{g}}$ of the density matrix that couples ground states to excited states, and it has eigenvalue -1 when operating on a part $P_{\mathrm{g}} \sigma P_{\mathrm{e}}$ that couples excited states to ground states. When we introduce the parameters

$$
\lambda_{k}=\int \mathrm{d} \eta w(\eta)\left(1-\mathrm{e}^{-\mathrm{i} \eta k}\right)
$$

the operators $W_{\mathrm{e}}$ and $W_{\mathrm{r}}$ can be specified by their action on an arbitrary density matrix $\sigma$, according to the relation

$$
\begin{aligned}
& W_{\mathrm{e}} \sigma=\lambda_{1} P_{\mathrm{g}} \sigma P_{\mathrm{e}}+\lambda_{-1} P_{\mathrm{e}} \sigma P_{\mathrm{g}} \\
& W_{\mathrm{r}} \sigma=\lambda_{1}\left(P_{\mathrm{e}} \sigma P_{\mathrm{e}}+P_{\mathrm{g}} \sigma P_{\mathrm{g}}\right)+\lambda_{2} P_{\mathrm{g}} \sigma P_{\mathrm{e}}
\end{aligned}
$$

Without loss of generality we can assume that the average increment in a phase jump is zero, since a non-zero average could be incorporated as a shifted frequency $\omega_{\mathrm{L}}$. Moreover, one would normally assume that $w(\eta)$ is an even function, which would make the parameters $\lambda_{k}$ real, and independent of the sign of $k$.

From the appendix it follows that

$$
\left\langle\exp \left[-\mathrm{i} k\left(\psi(t)-\psi\left(t^{\prime}\right)\right)\right]\right\rangle=\exp \left(-\lambda_{k}\left(t-t^{\prime}\right)\right)
$$

for $t>t^{\prime}$. Hence $\lambda_{1}$ determines the decay of the autocorrelation function of the electric field, and therefore $\lambda_{1}$ is the bandwidth (HWHM) of the incident radiation, which has a Lorentzian profile. Only the parameters $\lambda_{1}$ and $\lambda_{2}$ show up in the spectrum of resonance fluorescence.

The phase-diffusion model is recovered if we assume that the phase fluctuation is a Gaussian process, so that all cumulants higher than the second vanish (Stratonovich 1963, Van Kampen 1981). This means that all moments of $w(\eta)$ higher than the second vanish, which corresponds to the limit of very frequent, very small phase jumps. From (3.8) we then find

$$
\lambda_{k}=\frac{1}{2} k^{2} \int \mathrm{d} \eta w(\eta) \eta^{2}
$$

Hence in the Gaussian limit the two parameters $\lambda_{1}$ and $\lambda_{2}$ are related according to $\lambda_{2}=4 \lambda_{1}$, whereas in general these two parameters are independent.

\section{Binary-collision approximation}

In the previous section we obtained equation (3.5), which expresses the average correlation function in a non-stochastic form with effective decay operators $W_{\mathrm{r}}$ and $W_{\mathrm{e}}$. After substitution of this equality in equation (2.11), the resulting expression for the fluorescence spectrum has exactly the same structure as in the case of monochromatic irradiation. In the case that only binary atom-perturber collisions occur, the spectrum can be expressed entirely in operators acting on the density matrix of the atom alone, either by projection-operator techniques (Burnett and Cooper 1980), or 
by truncation of a BBGKY hierarchy (Nienhuis 1982). The result is

$I(\omega)=(a / \pi) \operatorname{Tr} d^{\dagger}\left[\Phi\left(\omega-\omega_{\mathrm{L}}\right)+W_{\mathrm{r}}+\Gamma+\mathrm{i} L_{\mathrm{d}}-\mathrm{i}\left(\omega-\omega_{\mathrm{L}}\right)\right]^{-1}\left(\bar{\sigma}_{0} d+C\left(\omega-\omega_{\mathrm{L}}\right) \bar{\sigma}_{0}\right)$.

The effect of the relaxation operator $\Phi$ on an atomic density matrix is

$$
\Phi\left(\omega-\omega_{\mathrm{L}}\right) \sigma_{0}=N \operatorname{Tr}_{1}\left(\omega-\omega_{\mathrm{L}}-L_{\mathrm{d}}\right) \frac{i}{\omega-\omega_{\mathrm{L}}-L_{\mathrm{d}}-L_{1}} L_{1} \rho_{\mathrm{p}}(1) \sigma_{0}
$$

where $\rho_{\mathrm{p}}(1)$ is the equilibrium density matrix of perturber 1 . The correlation operator $C$ describes the effect of fluorescent emission during a collision, and it is important only when $\omega-\omega_{L}$ has a value far from the eigenvalues of $L_{\mathrm{d}}$. It is defined by the equation

$C\left(\omega-\omega_{\mathrm{L}}\right) \sigma_{0}=-N \operatorname{Tr} L_{1} \frac{1}{\omega-\omega_{\mathrm{L}}-L_{\mathrm{d}}-L_{1}}\left[\left(\frac{1}{L_{\mathrm{d}}+L_{1}} L_{1} \rho_{\mathrm{p}}(1) \sigma_{0}\right) d\right]$.

An infinitesimal positive imaginary part should be included in $\omega$ for reasons of convergence. Strictly speaking, $L_{\mathrm{d}}$ should be read as $L_{\mathrm{d}}-\mathrm{i}\left(W_{\mathrm{r}}+\Gamma\right)$ in (4.2) and in the first denominator in (4.3), whereas in the second denominator $L_{\mathrm{d}}$ should be $L_{\mathrm{d}}-$ $\mathrm{i}\left(W_{\mathrm{e}}+\Gamma\right)$. However, in these single-collision operators the decay operators are effective only for the duration of a collision $\tau_{c}$, and when both the natural linewidth and the radiation bandwidth are small compared with the inverse duration of a collision, the decay operators $\Gamma$ and $W$ are negligible in (4.2) and (4.3). On the other hand, when the bandwidth is not small compared with $\tau_{c}^{-1}$, the effect of $W_{\mathrm{r}}$ in (4.1) is dominant over the effect of the relaxation operator $\Phi$, which is of the order of the collision frequency, and then collisional broadening effects in (4.1) are obscured altogether. We conclude that it is appropriate in all cases to use the expressions (4.2) and (4.3), which are identical as in the case of monochromatic irradiation.

For the steady-state reduced density matrix $\bar{\sigma}_{0}$ of the atom in (4.1) we must take the eigenvector with eigenvalue zero of the effective one-particle evolution operator $\Phi(0)+\Gamma+W_{\mathrm{e}}+\mathrm{i} L_{\mathrm{d}}$ (Nienhuis 1981).

The net effect of the phase fluctuations on the fluorescence spectrum is now twofold. Firstly, in the rate equation determining the steady-state density matrix $\bar{\sigma}_{0}$, the decay of coherence between the excited levels and the ground state is enhanced with the bandwidth $\lambda_{1}$. Hence also the integrated fluorescent intensity can only depend upon this bandwidth $\lambda_{1}$. Secondly, in the regression of the correlation function, which determines the spectral distribution, the diagonal part of the operator $\bar{U}\left(\bar{\sigma}_{0} d\right)$ gets an additional decay rate $\lambda_{1}$, and the decay of the part coupling the excited states to the ground states is enhanced with the term $\lambda_{2}$, according to equation (3.10).

\section{Two-state atom}

Virtually all papers so far dealing with finite-bandwidth effects in atomic optical resonance have ignored possible degeneracy of the atomic levels. The formal result of the previous section holds equally well for transitions between atomic multiplets of degenerate states. In this section we shall work out in more detail the fluorescence spectrum of a two-state atom, and we mainly follow the notation of two previous 
papers (Nienhuis 1981, 1982). Moreover, we are mainly interested in the spectrum in the neighbourhood $\left(\sim \tau_{c}^{-1}\right)$ of the transition frequencies of the dressed atom, where the effect of the correlation operator $C$ may be ignored.

\subsection{Modification in the fluorescence spectrum}

The various operators occurring in (4.1) can be represented as four-dimensional matrices in the case of a two-state atom. As usual (Reynaud and Cohen-Tannoudji 1982, Nienhuis 1.982) we choose the unit matrix and the three Pauli operators as basis

$$
\begin{array}{ll}
1=|\mathrm{e}\rangle\langle\mathrm{e}|+| \mathrm{g}\rangle\langle\mathrm{g}| & S_{x}=|\mathrm{e}\rangle\langle\mathrm{g}|+| \mathrm{g}\rangle\langle\mathrm{e}| \\
S_{\mathrm{y}}=-\mathrm{i}|\mathrm{e}\rangle\langle\mathrm{g}|+\mathrm{i}| \mathrm{g}\rangle\langle\mathrm{e}| & S_{z}=|\mathrm{e}\rangle\langle\mathrm{e}|-| \mathrm{g}\rangle\langle\mathrm{g}| .
\end{array}
$$

The relaxation operator may be assumed to have matrix elements only between the basis elements $S_{x}$ and $S_{y}$. The dressed-atom Hamiltonian $H_{\mathrm{d}}$ takes the form

$$
H_{\mathrm{d}}=-\frac{1}{2} \hbar\left(\Omega S_{x}+\Delta S_{z}\right)
$$

where $\Omega=d_{\mathrm{L}} E_{0} / \hbar$ is the Rabi frequency, and $\Delta=\omega_{\mathrm{L}}-\omega_{\mathrm{eg}}$ is the detuning of the central frequency from resonance.

The steady-state density matrix $\bar{\sigma}_{0}$ can be expanded as

$$
\overline{\boldsymbol{\sigma}}_{0}=\frac{1}{2}(1+\overline{\boldsymbol{P}} \cdot \boldsymbol{S})
$$

and it is an eigenvector of $\Phi(0)+\Gamma+W_{\mathrm{e}}+\mathrm{i} L_{\mathrm{d}}$ with eigenvalue zero. On the basis $(5.1)$ this operator has the matrix representation

$$
\Phi(0)+\Gamma+W_{\mathrm{e}}+\mathrm{i} L_{\mathrm{d}}=\left(\begin{array}{llll}
0 & 0 & 0 & 0 \\
\boldsymbol{b} & & \mathbf{G} &
\end{array}\right)
$$

where $b$ is a three-dimensional vector, and $\mathbf{G}$ is a three-dimensional matrix

$$
\boldsymbol{b}=\left(\begin{array}{c}
0 \\
0 \\
A
\end{array}\right) \quad \mathbf{G}=\left(\begin{array}{ccc}
\frac{1}{2} A+\Phi_{x x}(0)+\lambda_{1} & -\Delta+\Phi_{x y}(0) & 0 \\
\Delta+\Phi_{y x}(0) & \frac{1}{2} A+\Phi_{y y}(0)+\lambda_{1} & -\Omega \\
0 & \Omega & A
\end{array}\right)
$$

with $A$ the spontaneous emission rate.

The steady-state density matrix $\bar{\sigma}_{0}$ is obtained by substituting into (5.3)

$$
\overline{\boldsymbol{P}}=-\mathbf{G}^{-1} \cdot \boldsymbol{b} .
$$

In order to evaluate the spectrum (4.1), we also need the matrix representation

$$
\Phi(\Lambda)+W_{\mathrm{r}}+\Gamma+\mathrm{i} L_{\mathrm{d}}-\mathrm{i} \Lambda=\left(\begin{array}{cccc}
-\mathrm{i} \Lambda+\lambda_{1} & 0 & 0 & 0 \\
\boldsymbol{b} & & \mathbf{G}_{\mathrm{r}}(\Lambda) &
\end{array}\right)
$$

where $\Lambda=\omega-\omega_{\mathrm{L}}$, and where the three-dimensional regression matrix is

$$
\mathbf{G}_{\mathrm{r}}(\Lambda)=\left(\begin{array}{ccc}
\frac{1}{2} A+\Phi_{x x}(\Lambda)+\frac{1}{2} \lambda_{2}-\mathrm{i} \Lambda & -\Delta+\Phi_{x y}(\Lambda)+\frac{1}{2} \lambda_{2} & 0 \\
\Delta+\Phi_{y x}(\Lambda)-\frac{1}{2} \lambda_{2} & \frac{1}{2} A+\Phi_{y y}(\Lambda)+\frac{1}{2} \lambda_{2}-\mathrm{i} \Lambda & -\Omega \\
0 & \Omega & A+\lambda_{1}-\mathrm{i} \Lambda
\end{array}\right)
$$

The inversion of the matrix (5.7) can now be expressed in $\mathbf{G}_{\mathrm{r}}^{-1}$, and we obtain an expression for the fluorescence spectrum of a two-state atom in the form of matrix 
elements of $\mathbf{G}^{-1}$ and $\mathbf{G}_{\mathrm{r}}^{-1}$

$$
\begin{aligned}
I(\omega)=\frac{1}{4 \pi} \operatorname{Re}\left[\left(\bar{P}_{x}+\mathrm{i} \bar{P}_{y}\right) \frac{A+\lambda_{1}-\mathrm{i} \Lambda}{\mathrm{i} \Lambda-\lambda_{1}}(1-\mathrm{i} 0) \cdot \mathbf{G}_{\mathrm{r}}(\Lambda)^{-1} \cdot \boldsymbol{b}\right. \\
+A\left(1+\bar{P}_{z}\right)(1-\mathrm{i} \quad 0) \cdot \mathbf{G}_{\mathrm{r}}(\Lambda)^{-1} \cdot\left(\begin{array}{c}
1 \\
\mathrm{i} \\
0
\end{array}\right) .
\end{aligned}
$$

As in a previous paper (Nienhuis 1982) this result can be explicitly evaluated without difficulty, by substitution of (5.5)-(5.8), and the effect of the finite bandwidth is immediately tracked down. The resulting general expression is, however, not very illuminating except in some special cases, which we shall now consider.

\subsection{Limit of large bandwidth}

We consider the case where the bandwidth of the incident radiation is large compared with the natural and collisional linewidths, and therefore we assume that $\lambda_{1}, \lambda_{2} \gg A, \Phi$. One readily checks that then the first term in (5.9) is negligible compared with the second one.

When $\lambda_{1}$ is also large compared with $\Omega, \Delta, \Lambda$, then $\Omega$ must be small compared with $\tau_{c}^{-1}$, and we may substitute for the matrix elements of $\Phi$ their limits for $\Omega \rightarrow 0$ (Nienhuis 1982)

$$
\begin{aligned}
& \lim _{\Omega \rightarrow 0} \Phi_{x x}(\Lambda)=\lim _{\Omega \rightarrow 0} \Phi_{y y}(\Lambda)=\frac{1}{2}\left[\phi(\omega)+\phi^{*}\left(2 \omega_{L}-\omega\right)\right] \\
& \lim _{\Omega \rightarrow 0} \Phi_{y x}(\Lambda)=-\lim _{\Omega \rightarrow 0} \Phi_{x y}(\Lambda)=\frac{1}{2}\left[\phi(\omega)-\phi^{*}\left(2 \omega_{L}-\omega\right)\right]
\end{aligned}
$$

where $\phi(\omega)=\gamma(\omega)+\mathrm{i} \beta(\omega)$ with $\gamma$ and $\beta$ the frequency-dependent collisional width and shift, pertaining to the unified theory of line broadening. As an approximate expression for the fluorescence spectrum in this limit of large bandwidth we obtain

$I(\omega)=\left(A \Omega^{2} / 2 \pi\right) \operatorname{Re}\left[\left(\lambda_{1} A+\Omega^{2}\right)\left(\frac{1}{2} A+\phi(\omega)-\mathrm{i} \Lambda-\mathrm{i} \Delta+\Omega^{2} / 2 \lambda_{1}\right)\right]^{-1}$.

Saturation begins to occur when $\Omega^{2}$ becomes comparable with $\lambda_{1} A$. The spectrum (5.11) is a single spectral line at the same position as the low intensity absorption line. (Note that $\Lambda+\Delta=\omega-\omega_{\text {eg }}$.) The rate of stimulated transitions $\Omega^{2} / 2 \lambda_{1}$ enters as an additional radiative contribution to the linewidth.

When $\Omega$ and $\lambda_{1}$ have the same order of magnitude, and are both large compared with $A$ and $\Phi$, then collisional effects in the fluorescence spectrum become negligible, and we find the limiting expression

$$
\begin{aligned}
I(\omega)=(A / 4 \pi) & \operatorname{Re}\left[2\left(\lambda_{1}-\mathrm{i} \Lambda\right)\left(\lambda_{2}-\mathrm{i} \Lambda+\mathrm{i} \Delta\right)+\Omega^{2}\right] \\
& \times\left[\left(\frac{1}{2} \lambda_{2}-\mathrm{i} \Lambda\right) \Omega^{2}-\mathrm{i}(\Lambda+\Delta)\left(\lambda_{1}-\mathrm{i} \Lambda\right)\left(\lambda_{2}-\mathrm{i} \Lambda+\mathrm{i} \Delta\right)\right]^{-1} .
\end{aligned}
$$

This limiting expression is, of course, identical to the corresponding limit for free atoms (e.g. Kimble and Mandel 1977).

\subsection{Limit of well-separated lines}

For large values of the Rabi frequency $\Omega$ or of the detuning $\Delta$, the spectrum separates 
into three lines, at least for monochromatic incident radiation (Mollow 1969, 1977), and the separation between the lines amounts to

$$
\Omega^{\prime}=\Delta\left(1+\Omega^{2} / \Delta^{2}\right)^{1 / 2} .
$$

The strengths and the widths of the lines contain information on optical collisions, defined as collisional transitions between the eigenstates of the dressed-atom Hamiltonian (5.2) (Burnett et al 1982, Nienhuis 1982, Reynaud and Cohen-Tannoudji 1982). These eigenstates are

$$
|1\rangle=-|e\rangle \sin \frac{1}{2} \theta+|g\rangle \cos \frac{1}{2} \theta \quad|2\rangle=|e\rangle \cos \frac{1}{2} \theta+|g\rangle \sin \frac{1}{2} \theta
$$

with eigenvalues

$$
E_{1}=\frac{1}{2} \hbar \Omega^{\prime} \quad E_{2}=-\frac{1}{2} \hbar \Omega^{\prime} .
$$

The angle $\theta$ is defined by

$$
\tan \theta=\Omega / \Delta \quad-\frac{1}{2} \pi<\theta<\frac{1}{2} \pi .
$$

We now consider the case where $\Omega^{\prime}$ is the dominant parameter in the problem, and we require $\Omega^{\prime} \gg \lambda_{1}, A, \Phi$. Then the broadening due to collisions, radiative decay and the finite bandwidth are insufficient to cause appreciable overlap of the lines, and a separate measurement of their widths and intensities remains possible. For the theoretical evaluation of these quantities it is sufficient to assume that the steady-state density matrix $\bar{\sigma}_{0}$ is diagonal in the dressed states (5.14), and to ignore coupling between different eigenvectors of the dressed-atom Liouvillian $L_{\mathrm{d}}$ during the regression (Nienhuis 1982).

The steady-state density matrix $\bar{\sigma}_{0}$ in this limit is the eigenvector with eigenvalue zero of $\Phi(0)+\Gamma+W_{\mathrm{e}}+\mathrm{i} L_{\mathrm{d}}$, projected on the subspace spanned by $|2\rangle\langle 2|$ and $|1\rangle\langle 1|$. On this subspace, this operator has the matrix representation

with

$$
\left(\Phi(0)+\Gamma+W_{\mathrm{e}}+\mathrm{i} L_{\mathrm{d}}\right)_{\mathrm{r}}=\left(\begin{array}{rr}
p+r & -q-r \\
-p-r & q+r
\end{array}\right)
$$

$$
\begin{aligned}
& p=A[(1+\cos \theta) / 2]^{2}+k(\Omega, \Delta) \\
& q=A[(1-\cos \theta) / 2]^{2}+k(\Omega, \Delta) \\
& r=\frac{1}{2} \lambda_{1} \sin ^{2} \theta
\end{aligned}
$$

where

$$
k(\Omega, \Delta)=-\operatorname{Tr}|1\rangle\langle 1|\Phi(0)| 2\rangle\langle 2|
$$

is the rate of optical collisions. The steady-state density matrix $\bar{\sigma}_{0}$ is now

$$
\bar{\sigma}_{0}=(|2\rangle(q+r)\langle 2|+| 1\rangle(p+r)\langle 1|) /(p+q+2 r) .
$$

The finite bandwidth $\lambda_{1}$ tends to make the population of the dressed states more equal, and it has the same effect as an enhancement of the rate of optical collisions.

The Liouville operator $L_{d}$ has $|2\rangle\langle 1|$ as eigenvector with eigenvalue $-\Omega^{\prime},|1\rangle\langle 2|$ as eigenvector with eigenvalue $\Omega^{\prime}$, and the two eigenvectors $|2\rangle\langle 2|$ and $|1\rangle\langle 1|$ with eigenvalue zero. Each of these three eigenvalues gives rise to a line in the emitted spectrum, the fluorescence line at $\omega=\omega_{L}-\Omega^{\prime}$, the three-photon line at $\omega=\omega_{L}+\Omega^{\prime}$ and the Rayleigh line at $\omega=\omega_{\mathrm{L}}$ (Carlsten et al 1977, Courtens and Szöke 1977). The 
strengths and widths of these lines are found by projecting the regression operators in (4.1) or (5.9) on the eigenvectors corresponding to each one of these eigenvalues. The projected parts of $\Gamma$ and $\Phi$ are derived before (Nienhuis 1982), and the contribution of $W_{\mathrm{r}}$ is evaluated in the same fashion. On the subspace spanned by $|2\rangle\langle 2|$ and $|1\rangle\langle 1|$, which corresponds to the Rayleigh line, the regression operator can be taken as

$$
\left(\Phi(0)+\Gamma+W_{\mathrm{r}}+\mathrm{i} L_{\mathrm{d}}\right)_{r}=\left(\begin{array}{rr}
p+s & -q-t \\
-p-t & q+s
\end{array}\right)
$$

with $p$ and $q$ given by (5.18), and

$$
s=\lambda_{1}+\left(\lambda_{2}-2 \lambda_{1}\right) \sin ^{2} \theta / 4 \quad t=\left(\lambda_{2}-2 \lambda_{1}\right) \sin ^{2} \theta / 4 .
$$

The fluorescence line and the three-photon line have simple Lorentzian profiles, and the Rayleigh line is the sum of a coherent and an incoherent component, both of which are Lorentzian. The entire spectrum in the limit of large values of $\Omega^{\prime}$ is found to be

$$
\begin{gathered}
I(\omega)=\pi^{-1} S_{\mathrm{f}} \operatorname{Re}\left[\gamma_{f}+\mathrm{i} \beta_{\mathrm{f}}-\mathrm{i}\left(\omega-\omega_{\mathrm{L}}+\Omega^{\prime}\right)\right]^{-1}+\pi^{-1} S_{\mathrm{t}} \operatorname{Re}\left[\gamma_{\mathrm{t}}+\mathrm{i} \beta_{\mathrm{t}}-\mathrm{i}\left(\omega-\omega_{\mathrm{L}}-\Omega^{\prime}\right)\right]^{-1} \\
+\pi^{-1} S_{\mathrm{rc}} \operatorname{Re}\left[\gamma_{\mathrm{rc}}-\mathrm{i}\left(\omega-\omega_{\mathrm{L}}\right)\right]^{-1}+\pi^{-1} S_{\mathrm{ri}} \operatorname{Re}\left[\gamma_{\mathrm{ri}}-\mathrm{i}\left(\omega-\omega_{\mathrm{L}}\right)\right]^{-1}
\end{gathered}
$$

The line strengths are given by

$$
\begin{aligned}
& S_{\mathrm{f}}=\frac{A}{4} \frac{q+r}{p+q+2 r}(1+\cos \theta)^{2} \quad S_{\mathrm{t}}=\frac{A}{4} \frac{p+r}{p+q+2 r}(1-\cos \theta)^{2} \\
& S_{\mathrm{rc}}=\frac{1}{4} A \sin ^{2} \theta(p-q)^{2} /(p+q+2 r)(p+q+2 t) \\
& S_{\mathrm{ri}}=\frac{1}{2} A \sin ^{2} \theta[(p+t)(q+r)+(p+r)(q+t)] /(p+q+2 r)(p+q+2 t) .
\end{aligned}
$$

The linewidths and shifts are

$$
\begin{gathered}
\gamma_{\mathrm{f}}+\mathrm{i} \beta_{\mathrm{f}}=\Phi_{\mathrm{f}}+\frac{1}{4} A\left(2+\sin ^{2} \theta\right)+(1-\cos \theta) \lambda_{1}+\left[\frac{1}{2}(1-\cos \theta)\right]^{2}\left(\lambda_{2}-2 \lambda_{1}\right) \\
\gamma_{\mathrm{t}}+\mathrm{i} \beta_{\mathrm{t}}=\Phi_{\mathrm{t}}+\frac{1}{4} A\left(2+\sin ^{2} \theta\right)+(1+\cos \theta) \lambda_{1}+\left[\frac{1}{2}(1+\cos \theta)\right]^{2}\left(\lambda_{2}-2 \lambda_{1}\right) \\
\gamma_{\mathrm{rc}}=\lambda_{1} \quad \gamma_{\mathrm{ri}}=p+q+s+t .
\end{gathered}
$$

The collisional contribution to the width and shift of the fluorescence line and the three-photon line are

$$
\Phi_{\mathrm{f}}=\operatorname{Tr}|1\rangle\left\langle 2\left|\Phi\left(-\Omega^{\prime}\right)\right| 2\right\rangle\left\langle 1\left|\quad \Phi_{\mathrm{t}}=\Phi_{\mathrm{f}}^{*}=\operatorname{Tr}\right| 2\right\rangle\left\langle 1\left|\Phi\left(\Omega^{\prime}\right)\right| 1\right\rangle\langle 2| .
$$

For a semiclassical description of the collisions, these quantities acquire a simple expression in terms of matrix elements of the semiclassical scattering matrix (Nienhuis 1982).

The contributions of the finite bandwidth to the strengths, and the widths are contained in the parameters $r, s$ and $t$. The strengths $S_{\mathrm{f}}$ and $S_{\mathrm{t}}$ can be written in the form

$$
S_{\mathrm{f}}=\frac{1}{8} A(1+\cos \theta)^{2}(1-M) \quad S_{\mathrm{t}}=\frac{1}{8} A(1-\cos \theta)^{2}(1+M)
$$

with

$$
M=(p-q) /(p+q+2 r) .
$$

The finite-bandwidth effect diminishes $M$, thereby increasing the fluorescence strength 
$S_{\mathrm{f}}$ and decreasing the three-photon strength $S_{\mathrm{t}}$. The total strength of the Rayleigh line is

$$
S_{\mathrm{rc}}+S_{\mathrm{ri}}=\frac{1}{4} A \sin ^{2} \theta
$$

independent of the bandwidth and of collisions. However, increasing the bandwidth transfers intensity from the coherent component to the incoherent one, and also in this respect the finite-bandwidth effect resembles the effect of collisions. In the special case of the phase-diffusion model we have $\lambda_{2}=4 \lambda_{1}$, so that $r=t$. Then the quantity $M$ also determines $S_{\mathrm{rc}}$ and $S_{\mathrm{ri}}$, according to the relation

$$
S_{\mathrm{rc}}=\frac{1}{4} A \sin ^{2} \theta M^{2} \quad S_{\mathrm{ri}}=\frac{1}{4} A \sin ^{2} \theta\left(1-M^{2}\right)
$$

and the analogy between the effect of the finite bandwidth and of collisions on the line strengths is complete for the phase-diffusion model.

The effects on the linewidths are rather different. All linewidths are increased by a finite bandwidth. For moderate values of $\Omega / \Delta$, this increase is smaller for the fluorescence line than for the three-photon line. The coherent Rayleigh line has the same linewidth $\lambda_{1}$ as the incident radiation, as one would expect.

\section{Conclusions}

The effects of phase fluctuations of the driving radiation on the spectrum of resonance fluorescence can be formally incorporated by including effective decay operators $W_{\mathrm{r}}$ and $W_{\mathrm{e}}$ in the regression of the dipole correlation function and in the evolution of the average density matrix, as indicated in equations (3.3)-(3.6). This result holds equally well for free radiating atoms as for atoms in a bath of perturbers. The decay operator $W_{\mathrm{e}}$ depends only on the linewidth $\lambda_{1}$ of the incident radiation, and the operator $W_{r}$ also contains the parameter $\lambda_{2}$, as defined by (3.8). The binary-collision approximation of the formal result is obtained by standard techniques. The combined action of phase fluctuations and collisions is best illustrated for a two-state atom. In the case when the bandwidth is large compared with the collisional linewidth, the fluorescence spectrum contains a single line with a profile determined by the lowintensity frequency-dependent absorption linewidth, and by a power-broadening parameter. This result is expressed in equation (5.11). At very high intensities, when the Rabi frequency $\Omega$ is comparable with the bandwidth $\lambda$, the collisional broadening is completely masked, as indicated in equation (5.12).

Of more practical interest for collision studies is the case where the bandwidth of the incident radiation is small compared with the separation between the lines in the Mollow triplet. The dominant parts of these lines are then given by equation (5.23), and the strengths and the widths of these lines are affected by collisions as well as by the phase fluctuations. An increase of the bandwidth has the same effect on the line strengths as collisions leading to an increase of the fluorescence line, a decrease of the three-photon line, and transfer of intensity from the coherent Rayleigh line to the incoherent one. The asymmetry of the spectrum, which was already present as a result of collisions, is further enhanced by the finite bandwidth. When one wants to use the line intensities in order to determine the rate of optical collisions, it is essential to take the phase fluctuations into account.

On the other hand, the two broadening effects have a rather different result for the linewidths. The fluorescence line is much less broadened by the phase fluctuations 
than the three-photon line, whereas the collisional broadening of these two lines is the same. The coherent Rayleigh line reproduces the profile of the incident radiation, and the incoherent Rayleigh line is both broadened and strengthened by the phase fluctuations. The corrections on the linewidths due to the finite bandwidth are expressed as simple additive corrections in (5.25).

\section{Appendix}

We wish to prove the differential equation (3.3) for the average of an operator $V$ that obeys the stochastic differential equation (3.2). For a given initial value $\psi(0)=\psi_{0}$ of the phase we introduce the characteristic function of the phase increment:

$$
f_{k}(t)=\left\langle\exp \left[-\mathrm{i} k\left(\psi(t)-\psi_{0}\right)\right]\right\rangle=\int \mathrm{d} \psi P(\psi, t) \exp \left[-\mathrm{i} k\left(\psi-\psi_{0}\right)\right]
$$

where $P$ is the conditional distribution function with the initial condition $P(\psi, 0)=$ $\delta\left(\psi-\psi_{0}\right)$. After taking the time derivative of (A.1), and substitution of the master equation (3.1), we obtain

$$
(\mathrm{d} / \mathrm{d} t) f_{k}(t)=-\lambda_{k} f_{k}(t)
$$

with $\lambda_{k}$ given by (3.8). Since $f_{k}(0)=1$, the function $f_{k}$ is independent of the initial value $\psi_{0}$. Equation (3.11) then follows by a trivial time translation.

As a generalisation we introduce the functional

$$
F(t)=\left\langle\exp \left(-\mathrm{i} \int_{0}^{t} \mathrm{~d} s \dot{\psi}(s) k(s)\right)\right\rangle
$$

for an arbitrary function of time $k(t)$. For an infinitesimal time increment $\mathrm{d} t$ we then obtain

$$
F(t+\mathrm{d} t)=\left\langle\exp [-\mathrm{i}(\psi(t+\mathrm{d} t)-\psi(t)) k(t)] \exp \left(-\mathrm{i} \int_{0}^{t} \mathrm{~d} s \dot{\psi}(s) k(s)\right)\right\rangle .
$$

Since $\psi(t)$ is a Markov process by definition, and since the conditional average of the first exponential in (A.4) is independent of the initial value $\psi(t)$, we can write

$$
F(t+\mathrm{d} t)=\left(1-\mathrm{d} t \int \mathrm{d} \eta w(\eta)\left(1-\mathrm{e}^{-\mathrm{i} k(t) \eta}\right)\right) F(t)
$$

and the result for $F(t)$ is

$$
F(t)=\exp \left(-\int_{0}^{t} \mathrm{~d} s \int \mathrm{d} \eta w(\eta)\left(1-\mathrm{e}^{-\mathrm{i} k(s) \eta}\right)\right) .
$$

In order to prove (3.3), we first write the formal solution of (3.2) in the form

$$
V(t)=\mathrm{e}^{-\mathrm{i} L_{0} t} \theta \exp \left(-\mathrm{i} \int_{0}^{t} \mathrm{~d} s \dot{\psi}(s) \tilde{L}_{1}(s)\right) V_{0}
$$

with $\tilde{L_{1}}(s)=\exp \left(\mathrm{i} L_{0} s\right) L_{1} \exp \left(-\mathrm{i} L_{0} s\right)$, and $\theta$ the Dyson time-ordering operator. Since (A.6) holds for an arbitrary function $k(t)$, and since averaging and time ordering are 
commuting operations, the average of (A.7) takes the formal expression.

$$
\langle V(t)\rangle=\mathrm{e}^{-\mathrm{i} L_{0} t} \theta \exp \left(-\int_{0}^{t} \mathrm{~d} s \int \mathrm{d} \eta w(\eta)\left(1-\mathrm{e}^{-\mathrm{i} \tilde{L}(s) \eta}\right)\right)\left\langle V_{0}\right\rangle .
$$

Equation (3.3) follows after time differentiation of (A.8).

\section{References}

Agarwal G S 1976 Phys. Rev, Lett. 371383 1978 Phys. Rev. A 181490

Avan P and Cohen-Tannoudji 1977 J. Phys. B: At. Mol. Phys. 10155

Burnett K and Cooper J 1980 Phys. Rev. A 222044

Burnett K, Cooper J, Kleiber P D and Ben-Reuven A 1982 Phys. Rev. A 251345

Carlsten J L, Szöke A and Raymer M G 1977 Phys. Rev. A 151029

Chaturvedi S and Gardiner C W 1981 J. Phys. B: At. Mol. Phys. 141119

Courtens E and Szöke A 1977 Phys. Rev. A 151588

Eberly J H 1976 Phys. Rev. Lett. 371387

Georges A T and Dixit S N 1981 Phys. Rev. A 232580

Georges A T and Lambropoulos P 1979 Phys. Rev. A 20991

Kimble H J and Mandel L 1977 Phys. Rev. A 15689

Knight P L, Molander W A and Stroud C R Jr 1978 Phys. Rev. A 171547

Mollow B R 1969 Phys. Rev. 1881969 1977 Phys. Rev. A 151023

Nienhuis G 1981 J. Phys. B: At. Mol. Phys. 143117 1982 J. Phys. B: At. Mol. Phys. 15535

Nienhuis G and Schuller F 1977 Physica 92C 397

Omont A, Smith E W and Cooper J 1972 Astrophys. J. 175185

Reynaud S and Cohen-Tannoudji C 1982 J. Physique $\mathbf{4 3} 1021$

Salomaa R 1978 J. Phys. B: At. Mol. Phys. 113745

Stratonovich R L 1963 Topics in the Theory of Random Noise (New York: Gordon and Breach) Van Kampen N G 1981 Stochastic Processes in Physics and Chemistry (Amsterdam: North-Holland) Wódkiewicz K 1980 Phys. Lett. 77A 315

Zoller P 1977 J. Phys. B: At. Mol. Phys. 10 L231

- 1978a J. Phys. B: At. Mol. Phys. 11805

- 1978 b J. Phys. B: At. Mol. Phys. 112825

1979a Phys. Rev. A 191151

- 1979b Phys. Rev. A 201019

- 1982 J. Phys. B: At. Mol. Phys. 152911

Zoller P, Alber G and Salvador R 1981 Phys. Rev. A 24398

Zoller P and Ehlotzky F 1977 J. Phys. B: At. Mol. Phys. 103023 